\title{
Stock Market and Motion of a Variable Mass Spring
}

\author{
E. Canessa* \\ The Abdus Salam International Centre for Theoretical Physics, Trieste, Italy
}

\begin{abstract}
We establish an analogy between the motion of spring whose mass increases linearly with time and volatile stock markets dynamics within an economic model based on simple temporal demand and supply functions [J. Phys. A: Math. Gen. 33, 3637 (2000)]. The total system energy $E_{t}$ is shown to be proportional to a decreasing time dependent spring constant $k_{t}$. This model allows to derive $\log$-periodicity $\cos \left[\log \left(t-t_{c}\right)\right]$ on commodity prices and oscillations (surplus and shortages) in the level of stocks. We also made an attempt to connect these results to the Tsallis statistics parameter $q$ based on a possible force-entropy correlation [Physica A 341, 165 (2004)] and find that the Tsallis second entropic term $\sum_{i=1}^{W} p_{i}^{q} /(q-1)$ relates to the square of the demand (or supply) function.
\end{abstract}

PACS numbers: 05.45.Tp, 05.20.-y, 89.90+n, 89.75.-k, 02.50-r

\footnotetext{
*E-mail: canessae@ictp.it
} 
The interplay of demand and supply of reserves (and commodities) is primordial for the stability of the economy (and the market) in general. The latter should also relate to the existence of clear $\log$-periodic periods of the form $\cos \left[\log \left(t-t_{c}\right)\right]$ (or $\sin \left[\log \left(t-t_{c}\right)\right]$ ) that characterize the daily dynamics of some of the most important indices worldwide [1, 2, 3]. In this work we introduce a simple spring system gaining momentum from the surroundings in order to give further insight into the temporal log-periodic phenomenon. We develop a 'toy' model based on an analogy with mechanics that exhibits these oscillations. Furthermore an analogy with a standard economic model based on simple $t$-dependent demand and supply stock functions is presented [4]. We correlate this result to the Tsallis statistics parameter $q$ based on a possible force-entropy correlation [5].

The interest in log-periodicity is twofold. Firstly they enhance the fit quality to observed data with better precision than simple power laws by adjusting the (frequency, local minima and maxima of) oscillations. Secondly their real-time monitoring could, in principle, allow for an enhancement of predictions in different contexts [6]. At the theoretical level, it has been argued that that there is a close relation between log-periodicity and the renormalization group theory [7, 8]. Logarithm periodic patterns also emerge from percolation models when applying the concept of clusters to groups of investors acting collectively [9, 10]. Besides, log-periodicities can be a consequence of transient clusters induced by an entropy-like term that may reflect the amount of cooperative information carried by the state of a large stochastic system of different species [11]. Alternatively, a simple model was introduced in [12] based on the interplay between nonlinear positive feedback and reversal in the inertia to account for accelerating log-periodic oscillations. However, there is not yet a fundamental theory that can explain the universality of log-periodic motion on large time scales.

The restoring force $F$ that a spring exerts on a fluctuating mass $m_{t}$ resting on a frictionless surface and attached to one end of the spring is defined by Hooke's law

$$
F=\frac{d\left(m_{t} v\right)}{d t}=\left(\frac{d m_{t}}{d t}\right) v+m_{t}\left(\frac{d v}{d t}\right) \equiv-k x,
$$

where $x$ is the displacement from the spring equilibrium position $x_{0}$ at time $t_{0}, v=d x / d t$ is the system velocity and $k$ an spring constant. This law holds for small changes in lenght.

The differential equation describing the spring's motion can then be approximated as

$$
\left(\frac{d m_{t}}{d x}\right)\left(\frac{d x}{d t}\right)^{2}+m_{t}\left(\frac{d^{2} x}{d t^{2}}\right)=-k_{t} x
$$


where, in general, the "spring constant" may also change with time when momentum is gained from, or lost to, the surroundings (e.g., rain or sand droping from a hopper at rest [13, 14, 15]). Let us consider the simplest case in which the mass increases linearly with time

$$
m_{t}=m_{0}\left(\frac{t}{t_{0}}\right)
$$

such that the slope $d m_{t} / d t \equiv m_{0} / t_{0}>0$ is the constant rate at which mass is gain. This could relate the asymptotic behaviour of a brownian particle having a fluctuating mass [16]. In other words our mass coefficient satisfies

$$
\frac{1}{m_{t}}\left(\frac{d m_{t}}{d x}\right)=\frac{1}{t}\left(\frac{d t}{d x}\right)=(v t)^{-1} .
$$

The oppositive case in which the mass of the oscillator decreases lineraly with time has been experimentally studied in [17].

The addition of mass implies less resistance by a soft sprint (with a smaller $k$ ) and thereafter a reduction of the spring constant as a function of time. Let us assume then for simplicity

$$
k_{t}=k_{0}\left(\frac{t_{0}}{t}\right)
$$

The time dependent angular frequency $\omega_{t} \equiv \sqrt{k_{t} / m_{t}}$, which it has been shown to remain valid when the mass is variable [17], defines the constant angle

$$
\theta \equiv \omega_{t} \cdot t=\sqrt{\frac{k_{0}}{m_{0}}} t_{0}=\omega_{0} \cdot t_{0} \equiv \theta_{0}
$$

Therefore the spring's equation of motion in eq.(2) under these constraints reduces to

$$
t^{2}\left(\frac{d^{2} x}{d t^{2}}\right)+t\left(\frac{d x}{d t}\right)+\theta^{2} x=0
$$

For $t \geq t_{0} \neq 0$, the general solution has the form

$$
x(t)=x_{0} \sin \left[\theta \ln \left(\frac{t}{t_{0}}\right)\right]+x_{1} \cos \left[\theta \ln \left(\frac{t}{t_{0}}\right)\right],
$$

with $x_{0,1}$ dimension constants. It follows then that the system in question displays temporal logarithm periodic oscillations as a consequence of adding mass linearly with time.

Setting $x_{1}=0$ in eq. (8) for simplicity, the system velocity is found to display decreasing logarithm periodic oscillations such that

$$
v(t)=x_{0} w_{t} \cos \left[\theta \ln \left(\frac{t}{t_{0}}\right)\right]=\frac{\theta}{t} \sqrt{x_{0}^{2}-x^{2}(t)} .
$$


The maximum and minimum peaks occur at the exponentially decaying time values $t_{n}=$ $t_{0} \exp [-\pi / 2 \theta]$. Hence the angle $\theta$ drives the peak positions of the oscillations.

Substitution of eq.(9) into (4) gives

$$
\ln m_{t}=\frac{1}{\theta} \int \frac{d x}{\sqrt{x_{0}^{2}-x^{2}(t)}}+\text { const. }
$$

Since $\sin \alpha=\sin (2 \pi n+\alpha)$, we then have

$$
\ln \left(\frac{m_{t}}{m_{0}}\right)=\left(\frac{1}{\theta}\right) \sin ^{-1}\left\{\sin \left[\theta \ln \left(\frac{t}{t_{0}}\right)\right]\right\}=\ln \left(\frac{t}{t_{0}}\right),
$$

which means that $m$ grows up proportional to $t$ as initially assumed in eq.(3)).

Let us establish next an analogy between the motion of a variable mass spring in eq.(17) and the dynamics of stock markets within a simple economic model based on simple $t$ dependent demand $D(P)$ and supply $Q(P)$ functions for one commodity [4, 8, 11]. The market will be considered competitive so it self-organizes to determine the behaviour of the asset price $P$ at time $t$ (i.e., no individual producer can set his own desired price).

In a competitive market the price rate increases usually as a functional of the excess demand function $E(P)=D(P)-Q(P)$, such that $d P / d t \equiv f[E(P)]$ [18]. Since in general a commodity can be stored, then stocks of the commodity build up when the flow of output exceeds the flow of demand and vice-versa. The rate at which the level of stocks, $S$, changes can then be approximated as $d S / d t=Q(P)-D(P)$. Thus a price adjustment relation that takes into account deviations of the stock level $S$ above certain optimal level $S_{o}$ (to meet any demand reasonably quickly) is simply given by

$$
\frac{d P}{d t}=-\gamma \frac{d S}{d t}+\lambda\left(S_{o}-S\right)
$$

where $\gamma$ (i.e., the inverse of excess demand required to move prices by one unity [19]) and $\lambda$ are positive factors. For $\lambda>0$, prices increase when stock levels are low and raise when they are high (with respect to $S_{o}$ ). When $\lambda=0$, the price adjusts at a rate proportional to the rate at which stocks are either raising or running down.

For all asset prices $P(t)$, simple forms for the demanded and supplied quantities are usually postulated

$$
\begin{aligned}
& D(P)=d^{*}+d_{o}(t)\left(P-P^{*}\right), \\
& Q(P)=q^{*}+q_{o}(t)\left(P-P^{*}\right),
\end{aligned}
$$


where $d_{o}, q_{o}$ are temporal functions related to material costs, wage rate, etc and $P^{*}=P\left(t^{*}\right)$, $d^{*}=D\left(P^{*}\right), q^{*}=Q\left(P^{*}\right)$ are values at equilibrium.

To complete this simplest, economics model let us consider as in [18] that $S_{o}$ depends linearly on the demand function

$$
S_{o}(P)=\ell_{o}+\ell D(P) \quad ; \quad \frac{d S_{o}}{d t}=\ell\left(\frac{d D}{d t}\right),
$$

with $\ell_{o}$ and $\ell$ constants. Therefore, in equilibrium (where $\left.\frac{d P}{d t}\right|_{P^{*}}=0$ and $\left.\frac{d S}{d t}\right|_{S^{*}}=0$, so that demand equals supply $D\left(P^{*}\right)=Q\left(P^{*}\right)$ and $\left.S=S^{*}=S_{o}\right)$, we obtain $d^{*}-q^{*}=0$ and $S^{*}=\ell_{o}+\ell d^{*}$.

After some algebra, it can be shown that the second derivative of the price variations in eq.(12) then satisfies

$$
\frac{d^{2} P}{d t^{2}}+\left[\gamma \beta_{o}(t)-\lambda \ell d_{o}(t)\right]\left(\frac{d P}{d t}\right)+\left[\gamma\left(\frac{d q_{o}}{d t}\right)-(\gamma+\lambda \ell)\left(\frac{d d_{o}}{d t}\right)+\lambda \beta_{o}(t)\right]\left(P-P^{*}\right)=0,
$$

where $\beta_{o}(t) \equiv q_{o}(t)-d_{o}(t)$. Since, in general, $d_{o}<0$ and $q_{o}>0$ then these conditions gives $\beta_{o}(t)>0[4]$.

From a comparison between the motion equation of oscillating systems that gain momentum from the surroundings as discussed in eq.(7) and the price adjustment eq.(15) for one commodity, we readily identify

$$
\begin{aligned}
P(t)-P^{*} & \rightleftharpoons x(t), \\
\gamma \beta_{o}(t)-\lambda \ell d_{o}(t) & \rightleftharpoons \frac{1}{t}, \\
\gamma\left(\frac{d q_{o}}{d t}\right)-(\gamma+\lambda \ell)\left(\frac{d d_{o}}{d t}\right)+\lambda \beta_{o}(t) & \rightleftharpoons\left(\frac{\theta}{t}\right)^{2} .
\end{aligned}
$$

This means to have analogous displacements, an analogous spring constant (related to an analogous mass via the relation $\left.k_{t} m_{t}=k_{0} m_{0}\right)$ and an analogous frequency. The above also leads to these conditions for log-periodicity in financial systems

$$
\begin{aligned}
\lambda \ell d_{o}(t) & \rightleftharpoons \gamma \beta_{o}(t)-\frac{1}{t}, \\
\lambda \ell q_{o}(t) & \rightleftharpoons(\gamma+\lambda \ell) \beta_{o}(t)-\frac{1}{t} .
\end{aligned}
$$

Thus within our analogy, insight into the nature of the observed log-periodicy in stocks markets can be obtained from the new perspective of the demand and supply functions. The expressions for $D$ and $Q$ of eq.(13) in conjuction with the above conditions for $d_{o}(t)<0$ 
and $q_{o}(t)>0$, depict the fact that as price falls, the quantity demanded for a commodity can increase under temporal constrains based in one of the basic principles of economy. That is, the higher the price, the higher the profit, then the higher the supply.

For this class of systems we derive via eqs.(8) and (9) and $x_{1}=0$, the universal relation

$$
\left(\frac{x}{x_{0}}\right)^{2}+\left(\frac{v}{x_{0} w_{t}}\right)^{2}=1
$$

for all time $t$, and the total system energy becomes

$$
E_{t} \equiv U+T=\frac{1}{2} k_{t} x^{2}+\frac{1}{2} m_{t} v^{2}=\frac{1}{2} k_{t} x_{0}^{2}=\left(\frac{k_{t}}{k_{0}}\right) U_{0},
$$

where $U= \pm \int k x d x= \pm \frac{1}{2} k x^{2}$ is the potential energy stored in the (compressed or elongated) spring and $T$ is the kinetics energy. Hence $E_{t}$ is inversely proportional to $t$ (or proportional to $k_{t}$ ). This general result can help to understand the reported log-periodic behaviour. Since from eq.(6) we have that $w_{t}$ is proportional to the inverse of time, then the total energy reaches a minimum only at $t \rightarrow \infty$. Hence systems displaying log-periodic behaviour are far from equilibrium and unstable (i.e., volatility spanning over several months in the case of financial systems [20]). Let us conclude making an attempt to connect the variable mass spring model to Tsallis statistics [21]. This could be useful to gain new insights on the observed log periodicity in stock market out of equilibrium (around times of financial turmoil) and the non-extensive entropic index $q$.

We have recently introduced a statistical thermodynamic approach of moving particles forming an elastic body which leads to reveal molecular-mechanical properties of classical and nonextensive dynamical systems by assuming a simple multiplicative form for the probability $p_{i}=\mu_{i} \cdot \nu_{i}$ that the system is in the microstate $i(=1, \cdots, W)$, which satisfies the factorization $\sum_{i=1}^{W} p_{i}=\sum_{i=1}^{N} \mu_{i} \cdot \nu_{i} \equiv 1[5]$. Expressions then follow for the the Helmholtz free energy $A=E-T \mathcal{S}$, considering the temperature $T$ and volume $V$ to be independent variables, with $E$ the internal energy and $\mathcal{S}$ the entropy of the system

$$
\begin{aligned}
A / k_{B} T & =\sum_{i=1}^{W} \mu_{i} \cdot \nu_{i} \ln \mu_{i}, \\
E / k_{B} T & =-\sum_{i=1}^{W} \mu_{i} \cdot \nu_{i} \ln \nu_{i}, \\
\mathcal{S} / k_{B} & =-\sum_{i=1}^{W} \mu_{i} \cdot \nu_{i} \ln \mu_{i} \cdot \nu_{i},
\end{aligned}
$$


with $k_{B}$ the Boltzman constant. Since the moving particles are assumed to form an elastic body, then the system entropy can be correlated to a tensile force acting on the system

$$
\mathcal{F}=\left(\frac{\partial A}{\partial x}\right)_{T}=k_{B} T \frac{\partial}{\partial x}\left(\sum_{i=1}^{W} \mu_{i} \cdot \nu_{i} \ln \mu_{i}\right)_{T}=\left(\frac{\partial E}{\partial x}\right)_{T}-T\left(\frac{\partial \mathcal{S}}{\partial x}\right)_{T} .
$$

Tsallis statistics is derived assuming the thermal energy of the particles $k_{B} T$ to be proportional to their energy states $\epsilon$ by the nonextensivity (integer) factor $q-1$ for all the $i$-microstates and $q \neq 1$. This was shown to be equivalent to $\ln \nu_{i}=1 / 1-q$. Then it follows that $\mathcal{S} / k_{B} \approx\left[1-\sum_{i=1}^{W}\left(\mu_{i} \cdot \nu_{i}\right)^{q}\right] / q-1$, which corresponds to the entropy term introduced by Tsallis and, to a first approximation, a possible force-entropy correlation $\mathcal{F} / k_{B} T \approx \frac{\partial}{\partial x}\left(\sum_{i=1}^{W} p_{i}^{q} / q-1\right)_{T}$.

A comparison of the latter and eq.(19) for a system of variable mass springs gives

$$
-k_{t} x \rightleftharpoons k_{B} T \frac{\partial}{\partial x}\left(\frac{\sum_{i=1}^{W} p_{i}^{q}}{q-1}\right)_{T} .
$$

This implies that the Tsallis second entropic term relates directly to the square of demand (or supply) function given in eq.(13) and using eqs.(16), namely

$$
-\frac{1}{2}\left[\frac{k_{0} t_{0}}{d_{o}(t)}\left[\gamma \beta_{o}(t)-\lambda \ell d_{o}(t)\right]\left(D(P)-d^{*}\right)\right]^{2} \rightleftharpoons k_{B} T\left(\frac{\sum_{i=1}^{W} p_{i}^{q}}{q-1}\right)_{T} .
$$

This relation also provides some insight into possible temporal dynamics of the non-extensive systems and gives physical significance to the q-values.

In this way stock market turmoil (in terms of the behaviour of the demand and supply functions) belong to a class of oscillating systems that gain momentum from the surroundings and, in turn, may correlate to a class of non-extensive dynamical systems described by Tsallis statistics. We have considered both a variable mass and variable "spring constant". This interplay seems to be a necessary and sufficient condition to understand log-periodicity on commodity prices and oscillations like surplus and shortages in the level of stocks, relating prices adjustments, as for example in crude oil and petroleum products. Since, we have shown

$$
\frac{d S}{d t}=Q(P)-D(P) \propto P(t)-P^{*} \propto x(t) \propto \cos \left[\log \left(t-t_{c}\right)\right],
$$

then it is plausible that policy makers like the European Central Bank [22] could exploit the present results to handle demand/supply for (oil) reserves by looking at log-periodic oscillations [23]. When (oil) prices are high it is expensive to keep surplus of stock, whereas 
in the case of low prices it makes sense to accumulate stocks for future gains. This balance of the supply and demand defines about $80 \%$ of the resulting price (the difference is defined by speculation) [24].

[1] S. Drożdż, F. Ruf, J. Speth and M. Wójcik, Eur. Phys. J. B 10 (1999) 589.

[2] M. Bartolozzi et al., Int. J. Mod. Phys. C 16 (2005) 1347.

[3] S. Drożdż, G. Grummer and F. Ruf, J. Speth, Physica A 324 (2003) 174.

[4] E. Canessa, J. Phys. A: Math. Gen. 33, 3637 (2000).

[5] E. Canessa, Physica A 341 (2004) 165.

[6] D. Sornette, Proc. Nat. Acad. Sci. 99 (2002) 2522.

[7] D. Sornette, A. Johansen and J.-P. Bouchaud, J. Phys. I (France) 6 (1996) 167.

[8] E. Canessa, Eur. Phys. J. B 20 (2001) 591.

[9] D. Stauffer and D. Sornette, Physica A 252 (1998) 271.

[10] D. Stauffer and N. Jan, Physica A 277 (2000) 215.

[11] E. Canessa, J. Phys. A 33 (2000) 9131.

[12] D. Sornette and K. Ide, Int. J. Mod. Phys. C 14 (2003) 267.

[13] D. Halliday and R. Resnick, in "Physics -I,II" (Chap.9 , J. Wiley \& Sons, N.Y., 1978).

[14] C.A. Sousa, Eur. J. Phys. 23 (2002) 43.

[15] R.A. Diaz, D.L. Gonzalez, F. Marin and R. Martinez, Preprint arXiv:physics/0310010 v2, Oct 2003.

[16] M. Ausloos and R. Lambiotte, Phys. Rev. E 73 (2006) 011105.

[17] J. Flores, G. Solovey and S. Gil, Am. J. Phys. 71 (2003) 721.

[18] D.L. Clements, An Introduction to Mathematical Models in Economic Dynamics (North Oxford Academic, Oxford, 1984).

[19] J.P. Bouchaud and R. Cont, Eur. Phys. J. B 6 (1998) 543.

[20] N. Vandewalle et al., Physica A 255 (1998) 201; Eur. J. Phys. B 4 (1998) 139; Ibid Eur. J. Phys. B 4 (1999) 355.

[21] C. Tsallis, J. Stat. Phys. 52 (1988) 479.

[22] Committee on the Global Financial System (CGFS) Papers No 31, July 2008 -available at http://www.bis.org/publ/cgfs31.pdf 
[23] Energy information Administration (EIA) from the US Goverment. Website: www.eia.doe.gov

[24] G. Vygon and A. Kouchaev, Weekly Topic Institute of Financial Studies, Russia (2002). Website: www.ifs.ru 